\title{
CALCULATION METHOD OF HEAT CAPACITY CHANGE DURING ORGANIC COMPOUNDS VAPORIZATION AND SUBLIMATION
}

\author{
Lviv Polytechnic National University \\ 12 S.Bandery St., 79013 Lviv, Ukraine; phys.chem.Ip@gmail.com
}

Received: April 05, 2014 / Revised: J une 04, 2014 / Accepted: September 02, 2014

(C) Sobechko I., 2016

\begin{abstract}
On the basis of literature data the constancy of heat capacity change during vaporization $( \pm 2 \%)$ and sublimation $( \pm 8 \%)$ has been determined at $298 \mathrm{~K}$ for different organic compounds. The obtained equations allow to recalculate the enthalpy according to Kirchhoff's law easier compared with present techniques based on the increments method. The vaporization and sublimation enthalpies of 5-(2-nitrophenyl)-furan-2-carbaldehyde have been determined and recalculated for $298 \mathrm{~K}$ for the reliability proofing of the suggested method.
\end{abstract}

Keywords: heat capacity, phase transition, enthalpy, sublimation, vaporization, 5-(2-nitrophenyl)-furan-2carbaldehyde.

\section{Introduction}

Arylfurfurol derivatives are biologically active compounds. That is why they are used as the initial compounds during drugs synthesis [1]. The determination of their thermodynamic properties, namely sublimation and vaporization enthalpies, allows to solve some practical questions, optimize their purification and application, and extend the theoretical knowledge about their nature. Phase transfer enthalpies are determined by the experiments within the temperature range essentially differed from $298 \mathrm{~K}$ - the temperature at which they are represented in the reference books. Thus, the melting enthalpy is measured at the melting temperatures and the vaporization enthalpy is determined according to the temperature dependence of the saturated vapor pressure. The temperature ranges favorable for the investigations are various for different compounds and depend on their volatility. To recalculate these values for the standard ones it is necessary to know the heat capacity of the investigated compound under standard conditions.
The experimental measuring of heat capacity is very laborious and complicated method. Therefore the existing information about organic compounds heat capacity is very limited. Due to the same reason there are many attempts to solve the existing problem via empirical way, starting from the simplest one - calculation of the heat capacity relative to the atoms in the molecule and finishing by the modern method - relative to the increments $[2,3]$. Such increments allow to calculate the heat capacity of some organic compounds in solid, liquid and gaseous states with the error of $7-10 \%$. Two suggested equations [3] allow to calculate the heat capacity change during vaporization (Eq. (1)) and sublimation (Eq. (2)) of the organic compounds:

$$
\begin{aligned}
& \left(C_{p(l)}-C_{p(g)}\right)=10.58+0.26 \cdot C_{p(l), 298} \\
& \left(C_{p(s)}-C_{p(g)}\right)=0.75+0.15 \cdot C_{p(s), 298}
\end{aligned}
$$

where $C_{p(l, s, g), 298}$ - heat capacity of the compound in liquid, solid or gaseous states, respectively, at $298 \mathrm{~K}$.

The same characteristic during melting may be determined according to the difference between Eq. (2) and Eq. (1). In all cases the constants are determined relative to the increments typical of the examined molecule [3]. The given method has a series of shortcomings: the increment values are generalized and some of them are absent. These factors complicate the calculations, especially for the complex molecules.

The aim of this work is the search of more available method to calculate the above-mentioned values without using increments which are unknown for the majority of organic compounds. For this purpose we analyzed literature data containing information about heat capacity change during vaporization of different organic compounds at $298 \mathrm{~K}$. To check the adequacy of obtained regularities and compliance with the existing methods we 
show the experimental results concerning the temperature dependence of saturated vapor pressure of 5-(2nitrophenyl)-furan-2-carbaldehyde obtained via effusive method and recalculate the obtained values of enthalpies from the average temperatures to $298 \mathrm{~K}$.

\section{Experimental}

\subsection{Analysis of Heat Capacity Change at Phase Transitions}

For the statistical calculations we used molar heat capacities of different organic compounds (aliphatic, alicyclic and aromatic hydrocarbons; alcohols; ketones; aldehydes; acids; nitrogen-, fluorine- and chlorinecontaining compounds) in solid, liquid and gaseous aggregative states. These values were determined by different authors and published in the literature. Tables 1 and 2 represent the values of molar and specific heat capacity, as well as heat capacity change during vaporization (Table 1) and sublimation (Table 2). The standard deviation of the average value is calculated using the Student's coefficient ( $t$-criterion) for the significance level of 0.95 [4].

The reason under which we analyzed the specific values of this thermodynamic characteristic instead of usual molar heat capacity is the following. Intermolecular forces are determinative in the processes of aggregative transformations and they are provided by all atoms in the molecule. Therefore their quantity in the mass (volume) unit is more important factor than the amount of molecules formed by them. Thus, at $298 \mathrm{~K}$ vaporization enthalpy of decane 1 mole is close to the enthalpy of pentane 2 moles. Actually, in the homologous series of normal alkanes $\left(\mathrm{C}_{5}-\mathrm{C}_{17}\right)$ the specific vaporization enthalpies $\left(\Delta_{v a p} H_{298}\right)$ are almost the same $(362 \pm 2 \mathrm{~J} / \mathrm{g})$. The same situation but with other value $(324 \pm 6 \mathrm{~J} / \mathrm{g})$ is observed for branched 2,2-dimethylalkanes and 3,3,5,5tetramethylalkanes. The similar examples may be continued. It is logically to assume that the heat capacity change which is the first derivative of enthalpy change will preserve such property. Thus, the standard value of heat capacity in the liquid state for methanol is $81.1 \mathrm{~J} / \mathrm{mol} \cdot \mathrm{K}$; for hexanol $-243.2 \mathrm{~J} / \mathrm{mol} \cdot \mathrm{K}$; the specific values of heat capacity are 2.53 and $2.38 \mathrm{~J} / \mathrm{g} \cdot \mathrm{K}$, respectively.

Table 1

Change of standard heat capacity during organic compounds vaporization at $298 \mathrm{~K}$

\begin{tabular}{|c|c|c|c|c|c|c|c|}
\hline \multirow{2}{*}{ Formula } & \multirow{2}{*}{ Compound } & \multirow{2}{*}{$\begin{array}{l}\mathrm{MW}, \\
\mathrm{g} / \mathrm{mol}\end{array}$} & \multicolumn{2}{|c|}{$\mathrm{C}_{p(l)}, \mathrm{J} / \mathrm{K}$} & \multicolumn{2}{|c|}{$C_{p(g)}, \mathrm{J} / \mathrm{K}$} & \multirow{2}{*}{$\begin{array}{c}C_{p(g)}-C_{p(l)} \\
\mathrm{J} / \mathrm{g} \mathrm{K}\end{array}$} \\
\hline & & & $/ \mathrm{mol}$ & $/ \mathrm{g}$ & $/ \mathrm{mol}$ & $/ \mathrm{g}$ & \\
\hline 1 & 2 & 3 & 4 & 5 & 6 & 7 & 8 \\
\hline $\mathrm{C}_{2} \mathrm{H}_{4} \mathrm{O}$ & Ethanal & 44.05 & $89.1[5]$ & 2.021 & $55.3[5]$ & 1.256 & -0.765 \\
\hline $\mathrm{C}_{4} \mathrm{H}_{4} \mathrm{O}$ & Furan & 68.07 & $114.6[5]$ & 1.683 & $64.4[5]$ & 0.946 & -0.737 \\
\hline $\mathrm{C}_{4} \mathrm{H}_{6} \mathrm{O}$ & 2,3-Dihydrofuran & 70.09 & $121.1[6]$ & 1.728 & $72.3[7]$ & 1.031 & -0.697 \\
\hline $\mathrm{C}_{4} \mathrm{H}_{6} \mathrm{O}_{2}$ & Butyrolacton & 86.09 & $140.9[8]$ & 1.637 & $86.1[9]$ & 1.000 & -0.637 \\
\hline $\mathrm{C}_{4} \mathrm{H}_{6} \mathrm{O}_{3}$ & 4-Methyl-1,3-dioxolan-2-on & 102.09 & $167.4[5]$ & 1.640 & $107.6[5]$ & 1.054 & -0.586 \\
\hline $\mathrm{C}_{4} \mathrm{H}_{8}$ & 1-Butene & 56.11 & $129.0[10]$ & 2.299 & $85.6[11]$ & 1.525 & -0.774 \\
\hline $\mathrm{C}_{5} \mathrm{H}_{4} \mathrm{O}_{2}$ & Furfural & 96.08 & $162.8[13]$ & 1.694 & $98.1[5]$ & 1.021 & -0.673 \\
\hline $\mathrm{C}_{5} \mathrm{H}_{7} \mathrm{O}$ & 2-Methylfuran & 82.10 & $143.7[5]$ & 1.751 & $89.7[5]$ & 1.092 & -0.659 \\
\hline $\mathrm{C}_{5} \mathrm{H}_{10}$ & 1-Pentene & 70.13 & $154.9[14]$ & 2.209 & $108.2[11]$ & 1.543 & -0.666 \\
\hline $\mathrm{C}_{5} \mathrm{H}_{10}$ & Cyclopentane & 70.13 & $126.7[15]$ & 1.807 & $82.8[16]$ & 1.181 & -0.626 \\
\hline $\mathrm{C}_{5} \mathrm{H}_{10}$ & Diethylketone & 86.13 & $190.9[5]$ & 2.216 & $128.9[17]$ & 1.496 & -0.720 \\
\hline $\mathrm{C}_{5} \mathrm{H}_{10}$ & Methylpropylketone & 86.13 & $185.4[5]$ & 2.152 & $121.0[5]$ & 1.405 & -0.747 \\
\hline $\mathrm{C}_{5} \mathrm{H}_{10}$ & Pentanal & 86.13 & $174.4[5]$ & 2.025 & $122.6[5]$ & 1.423 & -0.602 \\
\hline $\mathrm{C}_{5} \mathrm{H}_{10}$ & iso-Propylmethylketone & 86.13 & $179.9[5]$ & 2.089 & 122.8 [17] & 1.425 & -0.664 \\
\hline $\mathrm{C}_{5} \mathrm{H}_{10}$ & Tetrahydrofuran & 86.13 & $149.6[18]$ & 1.737 & $99.1[19]$ & 1.151 & -0.586 \\
\hline $\mathrm{C}_{5} \mathrm{H}_{12}$ & Pentane & 72.15 & $167.2[20]$ & 2.317 & $120.1[21]$ & 1.664 & -0.653 \\
\hline $\mathrm{C}_{6} \mathrm{H}_{6}$ & Benzene & 78.11 & $135.6[22]$ & 1.736 & $82.4[11]$ & 1.055 & -0.681 \\
\hline $\mathrm{C}_{6} \mathrm{H}_{12}$ & Cyclopentane-methyl & 84.16 & $158.7[23]$ & 1.886 & $109.5[11]$ & 1.301 & -0.585 \\
\hline $\mathrm{C}_{6} \mathrm{H}_{12}$ & Cyclohexane & 84.16 & $156.9[22]$ & 1.864 & $105.3[16]$ & 1.251 & -0.613 \\
\hline $\mathrm{C}_{6} \mathrm{H}_{12} \mathrm{O}$ & iso-Butylmethylketone & 100.16 & $211.9[24]$ & 2.116 & $147.5[25]$ & 1.473 & -0.643 \\
\hline $\mathrm{C}_{6} \mathrm{H}_{12} \mathrm{O}$ & tert-Butylmethylketone & 100.16 & $206.9[5]$ & 2.066 & $157.1[25]$ & 1.568 & -0.498 \\
\hline
\end{tabular}


Table 1 (Continued)

\begin{tabular}{|c|c|c|c|c|c|c|c|}
\hline 1 & 2 & 3 & 4 & 5 & 6 & 7 & 8 \\
\hline $\mathrm{C}_{6} \mathrm{H}_{12} \mathrm{O}$ & Hexanal & 100.16 & $210.4[26]$ & 2.101 & $145.5[27]$ & 1.453 & -0.648 \\
\hline $\mathrm{C}_{6} \mathrm{H}_{12} \mathrm{O}$ & Methylbutylketone & 100.16 & $213.4[28]$ & 2.131 & $143.8[5]$ & 1.436 & -0.695 \\
\hline $\mathrm{C}_{6} \mathrm{H}_{12} \mathrm{O}$ & Propylethylketone & 100.16 & $216.9[5]$ & 2.166 & $151.0[5]$ & 1.508 & -0.658 \\
\hline $\mathrm{C}_{6} \mathrm{H}_{14}$ & Hexane & 86.18 & $197.6[29]$ & 2.293 & $142.6[27]$ & 1.655 & -0.638 \\
\hline $\mathrm{C}_{7} \mathrm{H}_{6} \mathrm{O}$ & Benzaldehyde & 106.12 & $172.0[5]$ & 1.621 & $111.7[5]$ & 1.153 & -0.568 \\
\hline $\mathrm{C}_{7} \mathrm{H}_{8} \mathrm{O}$ & Metoxybenzene & 108.14 & $199.0[30]$ & 1.840 & $135.3[5]$ & 1.251 & -0.589 \\
\hline $\mathrm{C}_{7} \mathrm{H}_{14}$ & Methylcyclohexane & 98.19 & $185.0[15]$ & 1.884 & $135.8[11]$ & 1.383 & -0.501 \\
\hline $\mathrm{C}_{7} \mathrm{H}_{14}$ & Cycloheptane & 98.19 & $180.5[31]$ & 1.838 & $132.0[16]$ & 1.344 & -0.494 \\
\hline $\mathrm{C}_{7} \mathrm{H}_{14} \mathrm{O}$ & Heptanal & 114.19 & $230.2[5]$ & 2.016 & $168.2[5]$ & 1.473 & -0.543 \\
\hline $\mathrm{C}_{7} \mathrm{H}_{16}$ & Heptane & 100.20 & $224.6[32]$ & 2.242 & $165.2[33]$ & 1.649 & -0.593 \\
\hline $\mathrm{C}_{7} \mathrm{H}_{16}$ & 2-Methylhexane & 100.20 & $222.9[34]$ & 2.225 & $165.4[33]$ & 1.651 & -0.574 \\
\hline $\mathrm{C}_{7} \mathrm{H}_{16} \mathrm{O}$ & Heptanol & 116.20 & $270.8[5]$ & 2.330 & $178.7[5]$ & 1.538 & -0.792 \\
\hline $\mathrm{C}_{8} \mathrm{H}_{10}$ & 1,2-Dimethylbenzene & 106.17 & $187.7[35]$ & 1.768 & $132.5[36]$ & 1.248 & -0.520 \\
\hline $\mathrm{C}_{8} \mathrm{H}_{10}$ & 1,3-Dimethylbenzene & 106.17 & $184.6[37]$ & 1.739 & $125.8[36]$ & 1.185 & -0.554 \\
\hline $\mathrm{C}_{8} \mathrm{H}_{10}$ & 1,4-Dimethylbenzene & 106.17 & $183.7[38]$ & 1.730 & $126.0[12]$ & 1.187 & -0.543 \\
\hline $\mathrm{C}_{8} \mathrm{H}_{16} \mathrm{O}$ & Hexamethylketone & 128.21 & $273.3[5]$ & 2.132 & $189.6[5]$ & 1.479 & -0.653 \\
\hline $\mathrm{C}_{8} \mathrm{H}_{16} \mathrm{O}$ & Octanal & 128.21 & $259.6[5]$ & 2.025 & $191.2[5]$ & 1.491 & -0.534 \\
\hline $\mathrm{C}_{8} \mathrm{H}_{18}$ & 2-Methylheptane & 114.23 & $252.0[39]$ & 2.206 & $187.2[33]$ & 1.639 & -0.567 \\
\hline $\mathrm{C}_{8} \mathrm{H}_{18}$ & Octane & 114.23 & $255.7[40]$ & 2.239 & $187.8[33]$ & 1.644 & -0.595 \\
\hline $\mathrm{C}_{8} \mathrm{H}_{18}$ & 2,3,4-Trimethylpentane & 114.23 & $247.3[41]$ & 2.165 & $191.6[33]$ & 1.677 & -0.488 \\
\hline $\mathrm{C}_{8} \mathrm{H}_{18} \mathrm{O}$ & Octanol & 130.23 & $304.0[5]$ & 2.334 & $201.5[5]$ & 1.548 & -0.787 \\
\hline $\mathrm{C}_{9} \mathrm{H}_{12}$ & Cumene & 120.19 & $215.4[42]$ & 1.792 & $151.7[27]$ & 1.262 & -0.530 \\
\hline $\mathrm{C}_{9} \mathrm{H}_{12}$ & Propylbenzene & 120.19 & $214.7[43]$ & 1.786 & $152.3[27]$ & 1.267 & -0.519 \\
\hline $\mathrm{C}_{9} \mathrm{H}_{12}$ & 1,2,3-Trimethylbenzene & 120.19 & $216.4[44]$ & 1.801 & $159.1[45]$ & 1.324 & -0.477 \\
\hline $\mathrm{C}_{9} \mathrm{H}_{12}$ & 1,2,4- Trimethylbenzene & 120.19 & $213.1[46]$ & 1.773 & $154.1[45]$ & 1.282 & -0.491 \\
\hline $\mathrm{C}_{9} \mathrm{H}_{12}$ & 1,3,5-Trimethylbenzene & 120.19 & $207.9[37]$ & 1.729 & $147.4[45]$ & 1.226 & -0.503 \\
\hline $\mathrm{C}_{9} \mathrm{H}_{18} \mathrm{O}$ & Nonanal & 142.24 & $290.3[5]$ & 2.041 & $214.1[5]$ & 1.505 & -0.536 \\
\hline $\mathrm{C}_{9} \mathrm{H}_{20}$ & 3,3-Diethylpentane & 128.26 & $278.2[47]$ & 2.169 & $216.7[33]$ & 1.690 & -0.479 \\
\hline $\mathrm{C}_{9} \mathrm{H}_{20}$ & 2,2,3,3,-Tetramethylpentane & 128.26 & $271.5[48]$ & 2.117 & $212.1[33]$ & 1.654 & -0.463 \\
\hline $\mathrm{C}_{10} \mathrm{H}_{8}$ & Naphthalene & 128.17 & $196.1[49]$ & 1.530 & $133.0[11]$ & 1.038 & -0.492 \\
\hline $\mathrm{C}_{10} \mathrm{H}_{14}$ & 1,2,3,4-Tetramethylbenzene & 134.22 & $244.3[50]$ & 1.820 & $186.1[45]$ & 1.387 & -0.433 \\
\hline $\mathrm{C}_{10} \mathrm{H}_{14}$ & 1,2,3,5- Tetramethylbenzene & 134.22 & $240.2[51]$ & 1.790 & $180.7[45]$ & 1.346 & -0.444 \\
\hline $\mathrm{C}_{10} \mathrm{H}_{22}$ & Decane & 142.28 & $315.5[40]$ & 2.217 & $233.1[33]$ & 1.638 & -0.579 \\
\hline $\mathrm{C}_{10} \mathrm{H}_{20} \mathrm{O}$ & Decanal & 156.27 & $319.7[5]$ & 2.046 & $237.0[5]$ & 1.517 & -0.529 \\
\hline $\mathrm{C}_{11} \mathrm{H}_{10}$ & 1-Methylnaphthaline & 142.20 & $224.4[53]$ & 1.578 & $159.3[11]$ & 1.120 & -0.458 \\
\hline $\mathrm{C}_{12} \mathrm{H}_{10}$ & Diphenyl & 154.21 & $259.5[54]$ & 1.683 & $166.7[55]$ & 1.081 & -0.602 \\
\hline $\mathrm{C}_{6} \mathrm{H}_{7} \mathrm{~N}$ & 2-Methylpyridine & 93.13 & $158.4[56]$ & 1.701 & $100.0[27]$ & 1.074 & -0.627 \\
\hline $\mathrm{C}_{6} \mathrm{H}_{7} \mathrm{~N}$ & 3-Methylpyridine & 93.13 & $158.7[57]$ & 1.704 & $99.6[27]$ & 1.070 & -0.634 \\
\hline $\mathrm{CH}_{3} \mathrm{NO}_{2}$ & Nitromethane & 61.04 & $106.0[58]$ & 1.736 & $57.3[27]$ & 0.939 & -0.797 \\
\hline $\mathrm{C}_{2} \mathrm{H}_{5} \mathrm{NO}_{2}$ & Nitroethane & 75.07 & $134.2[59]$ & 1.788 & $78.2[27]$ & 1.042 & -0.746 \\
\hline $\mathrm{C}_{2} \mathrm{H}_{4} \mathrm{~F}_{2}$ & 1,1-Difluoroethane & 66.05 & $118.4[60]$ & 1.793 & $67.9[27]$ & 1.028 & -0.765 \\
\hline $\mathrm{C}_{6} \mathrm{H}_{4} \mathrm{~F}_{2}$ & 1,2-Difluorobenzene & 114.09 & $159.0[61]$ & 1.394 & $106.5[27]$ & 0.933 & -0.461 \\
\hline $\mathrm{C}_{6} \mathrm{H}_{4} \mathrm{~F}_{2}$ & 1,3-Difluorobenzene & 114.09 & $159.1[62]$ & 1.395 & $106.3[27]$ & 0.932 & -0.463 \\
\hline $\mathrm{C}_{6} \mathrm{H}_{4} \mathrm{~F}_{2}$ & 1,4-Difluorobenzene & 114.09 & $159.1[63]$ & 1.394 & $106.9[27]$ & 0.937 & -0.457 \\
\hline $\mathrm{C}_{6} \mathrm{H}_{5} \mathrm{~F}$ & Fluorobenzene & 96.10 & $146.3[64]$ & 1.522 & $94.4[27]$ & 0.982 & -0.540 \\
\hline $\mathrm{C}_{7} \mathrm{H}_{5} \mathrm{~F}_{3}$ & Trifluoromethylbenzene & 146.11 & $188.5[65]$ & 1.290 & $130.4[27]$ & 0.892 & -0.398 \\
\hline $\mathrm{C}_{7} \mathrm{H}_{7} \mathrm{~F}$ & 1-Fluoro-4-methylbenzene & 110.13 & $173.7[66]$ & 1.577 & $116.2[27]$ & 1.055 & -0.522 \\
\hline $\mathrm{C}_{6} \mathrm{H}_{5} \mathrm{Cl}$ & Chlorobenzene & 112.56 & $152.1[67]$ & 1.351 & $98.0[27]$ & 0.871 & -0.480 \\
\hline
\end{tabular}


Table 2

Change of standard heat capacity during organic compounds vaporization at $298 \mathrm{~K}$

\begin{tabular}{|c|c|c|c|c|c|c|c|}
\hline \multirow{2}{*}{ Formula } & \multirow{2}{*}{ Compound } & \multirow{2}{*}{$\begin{array}{l}\text { MW, } \\
\mathrm{g} / \mathrm{mol}\end{array}$} & \multicolumn{2}{|c|}{$\mathrm{C}_{p(l)}, \mathrm{J} / \mathrm{K}$} & \multicolumn{2}{|c|}{$C_{p(g)}, \mathrm{J} / \mathrm{K}$} & \multirow{2}{*}{$\begin{array}{c}C_{p(g)}-C_{p(l)} \\
\mathrm{J} / \mathrm{g} \mathrm{K}\end{array}$} \\
\hline & & & $/ \mathrm{mol}$ & $\lg$ & $/ \mathrm{mol}$ & $/ \mathrm{g}$ & \\
\hline $\mathrm{C}_{2} \mathrm{H}_{2} \mathrm{O}_{4}$ & Oxalic acid & 90.04 & $105.9[5]$ & 1.176 & $85.0[57]$ & 0.944 & -0.232 \\
\hline $\mathrm{C}_{3} \mathrm{H}_{4} \mathrm{O}_{3}$ & 1,3-Dioxolan-2-on & 88.06 & $117.4[5]$ & 1.333 & $81.6[5]$ & 0.927 & -0.406 \\
\hline $\mathrm{C}_{3} \mathrm{H}_{6} \mathrm{O}_{3}$ & 1,3,5-Trioxane & 90.08 & $111.4[5]$ & 1.237 & $82.4[5]$ & 0.915 & -0.322 \\
\hline $\mathrm{C}_{6} \mathrm{H}_{6} \mathrm{O}$ & Phenol & 94.11 & $127.45[5]$ & 1.354 & $103.2[5]$ & 1.097 & -0.257 \\
\hline $\mathrm{C}_{7} \mathrm{H}_{6} \mathrm{O}_{2}$ & Benzoic acid & 122.12 & $146.8[5]$ & 1.202 & $103.5[5]$ & 0.847 & -0.355 \\
\hline $\mathrm{C}_{7} \mathrm{H}_{8} \mathrm{O}$ & 2-Methylphenol & 108.14 & $154.6[5]$ & 1.429 & $127.3[5]$ & 1.177 & -0.252 \\
\hline $\mathrm{C}_{7} \mathrm{H}_{8} \mathrm{O}$ & 4-Methylphenol & 108.14 & $150.3[5]$ & 1.389 & $125.0[5]$ & 1.156 & -0.233 \\
\hline $\mathrm{C}_{7} \mathrm{H}_{10}$ & 2-Norborner & 94.15 & $129.9[68]$ & 1.380 & 112.7 [69] & 1.197 & -0.183 \\
\hline $\mathrm{C}_{10} \mathrm{H}_{8}$ & Naphthalene & 128.17 & $165.7[55]$ & 1.293 & $133.0[11]$ & 1.038 & -0.255 \\
\hline $\mathrm{C}_{10} \mathrm{H}_{14}$ & 1,2,4,5-Tetramethylbenzene & 134.22 & $204.0[52]$ & 1.520 & $183.1[45]$ & 1.364 & -0.156 \\
\hline $\mathrm{C}_{11} \mathrm{H}_{16}$ & Pentamethylbenzene & 148.25 & $270.3[52]$ & 1.823 & $212.5[45]$ & 1.433 & -0.390 \\
\hline $\mathrm{C}_{12} \mathrm{H}_{10}$ & Diphenyl & 154.21 & $198.4[70]$ & 1.287 & $166.7[55]$ & 1.081 & -0.206 \\
\hline $\mathrm{C}_{12} \mathrm{H}_{12}$ & 1,8-Dimethylnaphthalene & 156.22 & $241.8[71]$ & 1.548 & $189.2[11]$ & 1.211 & -0.337 \\
\hline $\mathrm{C}_{12} \mathrm{H}_{12}$ & 2,3- Dimethylnaphthalene & 156.22 & $216.5[71]$ & 1.386 & $188.0[11]$ & 1.203 & -0.183 \\
\hline $\mathrm{C}_{12} \mathrm{H}_{12}$ & 2,6- Dimethylnaphthalene & 156.22 & $202.5[71]$ & 1.296 & $180.8[11]$ & 1.157 & -0.139 \\
\hline $\mathrm{C}_{12} \mathrm{H}_{12}$ & 2,7- Dimethylnaphthalene & 156.22 & $204.4[71]$ & 1.308 & $181.6[11]$ & 1.162 & -0.146 \\
\hline $\mathrm{C}_{13} \mathrm{H}_{28} \mathrm{O}$ & 1-Tridecanol & 200.36 & $378.0[72]$ & 1.887 & $315.9[5]$ & 1.577 & -0.310 \\
\hline $\mathrm{C}_{14} \mathrm{H}_{28} \mathrm{O}$ & Tetradecanone & 212.37 & $415.2[73]$ & 1.955 & $328.6[5]$ & 1.547 & -0.408 \\
\hline $\mathrm{C}_{14} \mathrm{H}_{30} \mathrm{O}$ & 1-Tetradecanol & 214.39 & $426.5[74]$ & 1.989 & $338.7[5]$ & 1.580 & -0.409 \\
\hline $\mathrm{C}_{15} \mathrm{H}_{30} \mathrm{O}$ & 2-Pentadecanone & 226.40 & $426.8[73]$ & 1.885 & $349.7[5]$ & 1.545 & -0.340 \\
\hline $\mathrm{C}_{15} \mathrm{H}_{32} \mathrm{O}$ & 1-Pentadecanol & 228.41 & $400.0[74]$ & 1.751 & $361.6[5]$ & 1.583 & -0.168 \\
\hline $\mathrm{C}_{16} \mathrm{H}_{34} \mathrm{O}$ & 1-Hexadecanol & 242.44 & $422.0[74]$ & 1.741 & $384.5[5]$ & 1.586 & -0.155 \\
\hline $\mathrm{C}_{18} \mathrm{H}_{38}$ & Octadecane & 254.49 & $485.6[75]$ & 1.908 & $417.6[27]$ & 1.641 & -0.267 \\
\hline $\mathrm{C}_{6} \mathrm{H}_{5} \mathrm{Cl}$ & Chlorbenzene & 112.56 & $120.0[76]$ & 1.066 & $98.0[27]$ & 0.871 & -0.195 \\
\hline $\mathrm{C}_{6} \mathrm{H}_{4} \mathrm{Cl}_{2}$ & 1,4-Dichlorbenzene & 147.00 & $147.8[77]$ & 1.005 & 113.9 [27] & 0.775 & -0.230 \\
\hline
\end{tabular}

Table 3

Effusion investigations of 5-(2-nitrophenyl)-furan-2-carbaldehyde

\begin{tabular}{|c|c|c|c|c|c|c|c|c|}
\hline Aggregative state & $T, \mathrm{~K}$ & $\Delta m_{e f} \cdot 10^{3}, \mathrm{~g}$ & $\tau \cdot 10^{3}, \mathrm{~s}$ & $p, \mathrm{~Pa}$ & $T, \mathrm{~K}$ & $\Delta m_{e f} \cdot 10^{3}, \mathrm{~g}$ & $\tau \cdot 10^{3}, \mathrm{~s}$ & $p, \mathrm{~Pa}$ \\
\hline \multirow{5}{*}{ Solid } & 346.6 & 0.90 & 10.81 & 0.108 & 354.9 & 0.90 & 5.42 & 0.219 \\
\cline { 2 - 9 } & 347.9 & 0.80 & 9.02 & 0.116 & 358.6 & 1.45 & 5.41 & 0.355 \\
\cline { 2 - 9 } & 351.2 & 0.90 & 7.21 & 0.164 & 361.5 & 1.75 & 5.41 & 0.431 \\
\cline { 2 - 9 } & 351.2 & 0.95 & 7.22 & 0.173 & 363.1 & 1.55 & 3.62 & 0.572 \\
\cline { 2 - 9 } & 353.1 & 1.10 & 7.22 & 0.201 & 363.2 & 1.30 & 3.62 & 0.480 \\
\cline { 2 - 9 } & 353.2 & 0.60 & 3.62 & 0.218 & 368.0 & 2.46 & 3.62 & 0.913 \\
\cline { 2 - 9 } & 353.4 & 0.90 & 5.43 & 0.218 & 368.0 & 2.55 & 3.62 & 0.947 \\
\hline \multirow{6}{*}{ Liquid } & 378.0 & 6.60 & 3.62 & 2.484 & 387.9 & 6.00 & 1.82 & 4.561 \\
\hline & 378.0 & 6.60 & 3.62 & 2.485 & 388.5 & 5.90 & 1.83 & 4.464 \\
\hline
\end{tabular}




\subsection{Synthesis, Confirmation of Identity and Determination of Saturated Vapor Pressure for 5-(2-N itrophenyl)-furan-2- carbaldehyde}

Under normal conditions 5-(2-nitrophenyl)-furan2-carbaldehyde is a crystalline substance with the melting temperature $T_{m}=368.3 \pm 1.0 \mathrm{~K}$ (determined by a capillary method):

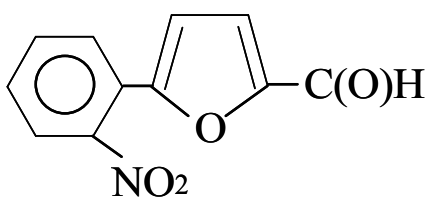

The investigated compound was obtained via interaction between furfural and arendiazonium chloride obtained by diazotation of 2-nitroaniline. The obtained precipitate was filtered and purified by multifold recrystallization in the mixture with ethanol dimethylformamide. The compound identity was confirmed by NMR-spectroscopy using Varian $600 \quad(600 \quad \mathrm{MHz})$ instrument and DMSO- $d 6$ solvent. The chemical shifts $(\delta, \mathrm{ppm})$ were recorded relative to DMSO signal $(2.5 \mathrm{ppm})$. ${ }^{1} \mathrm{H}$ NMR (600 MHz, DMSO) $\delta 7.24(\mathrm{~d}, J=3.7 \mathrm{~Hz}, 1 \mathrm{H}$, fur), $7.72(\mathrm{~d}, J=3.7 \mathrm{~Hz}, 1 \mathrm{H}$, fur), 7.79 (t, $J=7.8,1 \mathrm{H}$, $\left.\mathrm{C}_{6} \mathrm{H}_{4}\right), 7.88\left(\mathrm{t}, J=7.8 \mathrm{~Hz}, 1 \mathrm{H}, \mathrm{C}_{6} \mathrm{H}_{4}\right), 7.99(\mathrm{~d}, J=7.8,1 \mathrm{H}$, $\left.\mathrm{C}_{6} \mathrm{H}_{4}\right), 8.07$ (d, J=7.8 Hz, 1H, $\left.\mathrm{C}_{6} \mathrm{H}_{4}\right), 9.66(\mathrm{~s}, 1 \mathrm{H}, \mathrm{CHO})$.

Temperature dependence of the saturated vapor pressure, as well as sublimation and vaporization enthalpies were determined via Knudsen integral effusion method. The experimental plant and its reliability test are described in Ref. [78]. The weight of effunded compound $\left(\Delta m_{e f}\right)$ was determined by weightening of the effusion chamber with the accuracy of $\pm 5 \cdot 10^{-5} \mathrm{~g}$. The accuracy of temperature $(T)$ and effusion time $(\tau)$ measurements was $\pm 0.1 \mathrm{~K}$ and $\pm 10 \mathrm{~s}$, respectively. The hole diameter in membrane and its thickness were 0.591 and $0.050 \mathrm{~m}$, respectively. The results of effusion measurements including the saturated vapor pressure $(p)$, linear equation of temperature dependence of the saturated vapor pressure within the coordinates of the Clausius-Clapeyron equation and heat of the corresponding phase transitions are represented in Table 3.

\section{Results and Discussion}

While analyzing the standard heat capacities of the compounds in solid, liquid and gaseous states (Tables 1 and 2) we used the general procedure of statistical selection. At first we declined 12 compounds from 81 ones for the vaporization process (Table 2) and 4 compounds from 29 ones for the sublimation process (Table 3) because their characteristics differ from the average values by more than $\pm 2 \sigma$. At the second stage we analyzed the possible existence of the heat capacity change dependence during vaporization of different organic compounds. Alcohols and nitrocompounds have the value that is higher than the average one $C_{p(g)}-C_{p(l)}=$ $=0.78 \mathrm{~J} / \mathrm{g} \cdot \mathrm{K}$; aromatic, fluorine- and chlorine-derived compounds have a lower value $(0.49 \mathrm{~J} / \mathrm{g} \cdot \mathrm{K})$. It should be also noted that the number of analyzed compounds sometimes was miserable: e.g. for alcohols - 3; for nitrocompounds -2 ; for fluorine-derived -5 and for chlorine-derived -2 . The analysis using $t$-criterion confirms that these samples are statistically indistinguishable and may be treated as one generalized sample with average values and dispersions given below in Eqs. (3) and (4).

The results of statistical analysis show the consistency of heat capacity change during vaporization and sublimation at $298 \mathrm{~K}$ (Tables 1 and 2), according to which the molar heat capacity change may be determined according to the following equations:

$$
\begin{gathered}
\Delta_{v a p} C_{p_{298}}^{0}=\left(C_{p(g)}-C_{p(l)}\right)=-(0.591 \pm 0.024) \cdot M \\
\Delta_{s u b} C_{p_{298}}^{0}=\left(C_{p(g)}-C_{p(c)}\right)=-(0.261 \pm 0.035) \cdot M
\end{gathered}
$$

To check the suggested equations we recalculated the experimental values of sublimation and vaporization enthalpies obtained within $p-T$ range for $298 \mathrm{~K}$ using the Kirchhoff's equation and then compared them.

$$
\Delta H_{298}=\Delta H_{T}-\int_{298}^{T} \Delta C_{p} d T
$$

where $\Delta H_{T}$ - enthalpy of phase transition determined at the average temperature of investigated $p-T$ interval; $\Delta C_{p}$ - heat capacity change at phase transition

For the comparison we used sublimation and vaporization enthalpies determined by the calorimetric method using reference substances at $298 \mathrm{~K}$ (Table 4) and vaporization enthalpies obtained by $p-T$ measurements and recalculated using the Kirchhoff's equation and known values of heat capacity in condensed and gaseous states at $298 \mathrm{~K}$.

Vaporization enthalpies of nonane, decane, decanol-1 and 1,4-dimethylbenzene, as well as sublimation enthalpies of benzoic acid and bicycle[2,2,2] octane were obtained by the calorimetric method at $298 \mathrm{~K}$. It is impossible to determine the sublimation enthalpies of nonane, decane, and decanol-1 at $298 \mathrm{~K}$ because their melting temperatures are lower than the mentioned one. The given values were calculated using sublimation values obtained by the experiments and recalculated to the standard values using heat capacities of the compounds in solid and gaseous states. 
Comparison of vaporization enthalpies at $298 \mathrm{~K}$ calculated by known equations (1) and (2) and suggested ones (3) and (4) with experimental values

\begin{tabular}{|c|c|c|c|c|c|c|}
\hline \multirow{3}{*}{ Compound } & \multicolumn{3}{|c|}{$\Delta_{\text {vap }} H_{298}, \mathrm{~kJ} / \mathrm{mol}$} & \multicolumn{3}{|c|}{$\Delta_{s u b} H_{298}, \mathrm{~kJ} / \mathrm{mol}$} \\
\hline & \multirow[t]{2}{*}{ Exp. } & \multicolumn{2}{|c|}{$\begin{array}{l}\text { Eq., according to which } \\
\Delta C_{p} \text { is calcl. }\end{array}$} & \multirow[t]{2}{*}{ Exp. } & \multicolumn{2}{|c|}{$\begin{array}{l}\text { Eq., according to } \\
\text { which } \Delta C_{p} \text { is calcl. }\end{array}$} \\
\hline & & (1) & (3) & & (2) & (4) \\
\hline Nonane & $46.43[79]$ & 47.8 & 46.5 & $71.4[80]$ & 71.4 & 71.9 \\
\hline Decane & $51.4 \pm 0.1[79]$ & 52.9 & 51.3 & $82.4[80]$ & 82.4 & 82.8 \\
\hline Decanol-1 & $81.5 \pm 0.8[3]$ & 80.4 & 80.5 & $112.5 \pm 6.3[81]$ & 114.3 & 114.3 \\
\hline Benzoic acid & & & & $89.5 \pm 1.8[82]$ & 88.4 & 88.7 \\
\hline Bicyclo[2,2,2]octane & & & & $48 \pm 2[83]$ & & 47.6 \\
\hline 1,4-Dimethylbenzene & $42.4 \pm 0.1[3]$ & 43.1 & 42.8 & & & \\
\hline $\begin{array}{l}\text { 5-(2-Nitrophenyl)-furan-2- } \\
\text { carbaldehyde }\end{array}$ & - & 78.2 & 80.8 & - & 107.8 & 108.2 \\
\hline
\end{tabular}

While comparing the experimental values calculated in accordance with known Eqs. (1) and (2) and suggested ones (3) and (4) we observe the similarity of the obtained results; the difference is within experimental and calculation errors. Table 4 also represents the comparison of enthalpies of the investigated aldehyde recalculated by different methods.

Taking into account all mentioned above, one can see that the known complicated calculation methods have no essential advantages compared with the simpler method suggested by us.

\section{Conclusions}

The recalculation of vaporization and sublimation enthalpies for $298 \mathrm{~K}$ using the suggested simple equations allows to obtain the results with the error of $2-8 \%$. It is tantamount to the application of existed additive methods based on increments principle. Vaporization and sublimation enthalpies of 5-(2-nitrophenyl)-furan-2carbaldehyde were experimentally determined within the temperature range when the compound is in solid and liquid aggregative states. The adequacy of proposed method with the existing calculations is confirmed. The suggested Eqs. (3) and (4) have only empirical character. Therefore their application while extrapolation calculations for the compounds with the molecular weight over $250 \mathrm{~g} / \mathrm{mol}$ and phase transition temperature over $550 \mathrm{~K}$ is limited.

\section{Acknowledgments}

The author thanks Dr. Y.Horak for the given sample of 5-(2-nitrophenyl)-furan-2-carbaldehyde and post-graduated students A. Marshalek and M. Punyak for their assistance during effusive investigations.

\section{References}

[1] Karateev A., Koryagin A., Litvinov D. et al.: Chem. Chem. Technol., 2008, 1, 19.

[2] Ravdel A. and Ponomareva A. (Eds.): Kratkiy Spravochnyk Physico-Khimicheskykh Velichin. Khimiya, Leningrad 1983.

[3] Chickos J. and Acree W.: J. Phys. Chem. Ref. Data, 2003, 32, 519.

[4] Charykov A.: Matematicheskaya Obrabotka Resultatov Khimicheskogo Analiza. Khimiya, Leningrad 1984.

[5] Vasiliev I. and Petrov V.: Termodinamicheskie Svoistva Kislorod-soderzhashikh Soedineniy. Khimiya, Leningrad 1984.

「6] Steele W., Chirico R., Nguyen A. et al.: AIChE Symp. Ser., $1989, \mathbf{8 5}, 140$.

[7] Klots T.: Spectrochim. Acta, 1994, A50, 1725.

[8] Ismailov T. and Gabzalilova N.: Uzb. Khim. Zh., 1988, 48.

[9] Kudchadker S.: Thermochim. Acta, 1975, 12, 11.

「10ך Takeda K., Yamamuro O. and Suga H.: J. Phys. Chem. Solids, 1991, 22, 607.

[11] Thermodynamics Research Center, Texas A\&M University, College Station, Texas 1997.

[12] Chao J.: J. Phys. Chem. Ref. Data, 1986, 15, 1369.

[13] Omel'chenko F.: Izv. Vyssh. Ucheb. Zaved. Pishch. Tekhnol., 1962, 2, 151

[14] Messerly J., Todd S., Finke H. et al.: J. Chem. Thermodyn., 1990, 22, 1107.

[15] Tanaka R.: J. Chem. Eng. Data, 1985, 30, 267.

[16] Dorofeeva O.: J. Phys. Chem. Ref. Data, 1986, 15, 437.

[17] Hales J.: Trans. Faraday Soc., 1967, 63, 1876.

[18] Inglese A., Grolier J.-P. and Wilhelm E.: Fluid Phase Equilibr., 1984, 15, 287.

[19] Dorofeeva O.: Thermochim. Acta, 1992, 200, 121.

[20] Messerly J., Guthrie G., Todd S. et al: J. Chem. Eng. Data, $1967,12,338$.

[21] Kharin V.: Izv. Vyssh. Ucheb. Zaved., Neft. Gaz, 1985, 28, 63. [22] Lainez A., Rodrigo M., Wilhelm E. et al.: J. Chem. Eng. Data, 1989, 34, 332.

[23] Douslin D. and Huffman H.: J. Am. Chem. Soc., 1946, 68, 173. ¡24ך Vesely F., Barcal P., Zabransky M. et al.: Collect. Czech. Chem. Commun., 1989, 54, 602.

[25] Von Geiseler G.: Z. Phys. Chem. (Leipzig), 1973, 252, 170.

[26] Vasil'ev V., Bykova T. and Lebedev B.: Zh. Fiz. Khim., 1991, 65, 51 . 
[27] Stull D., Westrum E. and Sinke G.: The Chemical Thermodynamics of Organic Compounds. John Wiley \& Sons. New York-London 1969.

[281 Harrop D., Head A. and Lewis G.: J. Chem. Thermodyn., 1970, $2,203$.

[29] Andreoli-Ball L., Patterson D., Costas M. et al.: J. Chem. Soc. Faraday Trans., 1988, 84, 3991.

[30] Fenwick J., Harrop D. and Head A.: J. Chem. Thermodyn., 1975, 7, 944.

[31] Jolicoeur C., Boileau J., Bazinet S. et al.: Can. J. Chem., 1975, 53, 716.

[32] Tanaka R.: J. Chem. Eng. Data, 1987, 32, 176.

[33] Scott D.: J. Chem. Phys., 1974, 60, 3144.

¡34ך Huffman H., Gross M., Scott D. et al.: J. Phys. Chem., 1961, 65, 495 .

[35] Fortier J.-L. and Benson G.: J. Chem. Eng. Data, 1979, 24, 34.

[36] Draeger J.: J. Chem. Thermodyn., 1985, 17, 263.

[37] Grolier J.-P., Roux-Desgranges G., Berkane M. et al.: J. Chem. Thermodyn., 1993, 25, 41.

[381 Tardajos G., Aicart E., Costas M. et al.: J. Chem. Soc. Faraday Trans., 1986, 82, 2977.

[39] Messerly J. and Finke H.: J. Chem. Thermodyn., 1971, 3, 675.

[40] Andreoli-Ball L., Patterson D., Costas M. et al.: J. Chem. Soc., Faraday Trans. 1988, 84, 3991.

[41] Osborne N. and Ginnings D.: J. Res. NBS, 1947, 39, 453.

[42] Kishimoto K., Suga H. and Syuzo S.: Bull. Chem. Soc. Japan, 1973, 46, 3020.

[43] Messerly J., Todd S. and Finke H.: J. Phys. Chem., 1965, 69, 4304.

[44] Taylor R., Johnson B. and Kilpatrick J.: J. Chem. Phys., 1955, 23, 1225 .

[45] Draeger J.: J. Chem. Thermodyn., 1985, 17, 263.

[46] Wilhelm E., Inglese A., Roux A. et al.: Fluid Phase Equilibr., 1987, 34, 49 .

[47] Fuchs R. and Peacock L.: Can. J. Chem., 1979, 57, 2302.

[48] Finke H., Messerly J. and Douslin D.: J. Chem. Thermodyn., 1976, 8, 965.

[49] Chirico R., Knipmeyer S., Nguyen A. et al.: J. Chem. Thermodyn., 1993, 25, 1461.

[50] Kurbatov V.: Zhur. Obshch. Khim., 1947, 17, 1999.

[51] Huffman H., Parks G. and Barmore M.: J. Am. Chem. Soc., 1931, 53, 3876.

[52] Colomina M., Jimenez P., Roux M. et al.: J. Chem. Thermodyn., 1989, 21, 275.

[53] McCullough J., Finke H., Messerly J. et al.: J. Phys. Chem., 1957, 61, 1105.

[54] Newton R., Kaura B. and DeVries T.: Ind. Eng. Chem., 1931, 23, 35 .

[55] Dorofeeva O.: Unpubl. results. Thermocenter of Rus. Acad. of Science, Moskva 1997.

[56] Scott D., Hubbard W., Messerly J. et al.: J. Phys. Chem., 1963, 67, 680 .

[57] Scott D., Good W., Guthrie G. et al.: J. Phys. Chem., 1963, 67, 685.

[58] Jones W. and Giauque W.: J. Am. Chem. Soc., 1947, 69, 983.

[59] Liu K. and Ziegler W.: J. Chem. Eng. Data, 1966, 11, 187.

[60] Porichanskii E., Ponomareva O. and Svetlichnyi P.: Izv. Vyssh. Uchebn. Zaved., Energ., 1982, 3, 122.
[61] Scott D., Messerly J., Todd S. et al.: J. Chem. Phys., 1963, 38, 532.

[62] Messerly J. and Finke H.: J. Chem. Thermodyn., 1970, 2 , 867.

[63] Lichanot A.: Thermochim. Acta, 1991, 177, 265.

[64] Roux A., Grolier J.-P., Inglese A. et al.: Ber. Bunsenges. Phys. Chem., 1984, 88, 986.

[65] Scott D., Douslin D., Messerly J. et al:: J. Am. Chem. Soc., 1959, 81, 1015.

[66] Meva'a L. and Lichanot A.: Thermochim. Acta, 1990, 158, 335.

[67] Shehatta I.: Thermochim. Acta, 1993, 213, 1.

[68] Steele W.: J. Chem. Thermodyn., 1978, 10, 919

[69] Walsh R.: J. Chem. Thermodyn., 1975, 7, 149.

¡70] Chirico R., Knipmeyer S., Nguyen A. et al.: J. Chem. Thermodyn., 1989, 21, 1307.

[71] Good W.: J. Chem. Thermodyn., 1973, 5, 715.

[72] Mosselman C. and Dekker H.: J. Chem. Soc. Faraday Trans., 1975, 1, 417.

「73] Sunner S., Svensson Ch. and Zelepuga A.: J. Chem. Thermodyn., 1979, 11, 491.

[74] Mosselman C., Mourik J. and Dekker H.: J. Chem. Thermodyn., 1974, 6, 477.

[75]Messerly J., Guthrie G., Todd S. et al.: J. Chem. Eng. Data, 1967, 12, 338.

[76] Andrews D. and Haworth E.: J. Am. Chem. Soc., 1928, 50, 2998.

[77] Dworkin A., Figuiere P., Ghelfenstein M. et al.: J. Chem. Thermodyn., 1976, 8, 835.

[78] Krasulin A., Kozyro A. and Kabo G.: Zh. Prykl. Khim., 1987, 1, 104.

[79] Majer V. and Svoboda V.: Enthalpies of Vaporization of Organic Compounds. A Critical Review and Data Compilation. Blackwell Sci. Publ. Oxford 1985.

[80] Bondi A.: J. Chem. Eng. Data, 1963, 8, 371.

[81] Karnes H., Kybett B., Wilson M. et al.: J. Amer. Chem. Soc., 1965, 87, 5554.

[82] Dibrivny V., Van-Chin-Syan Yu. and Melnyk G.: Chem. Chem. Technol., 2008, 1, 1.

[83] Westrum E., Wong W. and Morawetz E.: J. Phys. Chem., 1970, 74, 2542.

\section{МЕТОД РОЗРАХУНКУ ЗМІНИ ТЕПЛОСМНОСТІ ПРИ ВИПАРОВУВАННІ ТА СУБЛІМАЦЇ ОРГАНІЧНИХ СПОЛУК}

Анотація. 3 а результатами аналізу джерел літератури встановлено постійність зміни теплоємності за $298 \mathrm{~K}$ при випаровуванні ( $\pm 2 \%)$ та сублімащії ( $\pm 8 \%$ ор органічних сполук різних класів. Одержані рівняння дають можливість проводити простіший перерахунок ентальпій за рівнянням Кірхгофа порівняно з існуючими методами, побудованими за принципом групових внесків. Для перевірки надійності запропонованого метода вперше визначено ентальпії випаровування та сублімаиії 5-(2-нітрофеніл)-фуран-2-карбальдегіда та проведено відповідні перерахунки на 298 K.

Ключові слова: теплоємність фазових переходів, ентальпії сублімачії та випаровування, 5-(2-нітрофеніл)-фуран2-карбальдегід. 
